\title{
Operative management options for symptomatic flexible adult acquired flatfoot deformity: a review
}

\author{
Htwe Zaw · James D. F. Calder
}

Published online: 5 January 2010

(C) Springer-Verlag 2009

\section{Introduction}

The treatment of adult acquired flatfoot deformity (AAFD) remains a complex clinical challenge. The vast array of surgical options available for the "flexible flatfoot", or stage II posterior tibial tendon dysfunction as classified by Johnson and Strom [30], reflects the wide variation in deformity and pathology seen in this group. This includes medial ray hypermobility, forefoot abduction and forefoot supination. We aimed at discussing the pathophysiology of AAFD, classification of the deformity and treatment options available with emphasis on the apparent surgical controversies in stage II disease.

\section{Pathophysiology}

Multiple factors are associated with the development of AAFD, but the most common is posterior tibial tendon dysfunction (PTTD). The tendon is an important dynamic arch stabilizer and a powerful invertor of the foot. It acts to "lock-in" the talonavicular joint and allows the triceps

H. Zaw · J. D. F. Calder

Department of Trauma and Orthopaedic Surgery,

Basingstoke and North Hampshire Hospitals

NHS Foundation Trust, Aldermaston Road,

Basingstoke RG24 9NA, UK

J. D. F. Calder

Imperial College School of Medicine Science and Technology,

Charing Cross Hospital, London, UK

H. Zaw (ه)

50 Copers Cope Road (Flat 3), Copers Cope Road,

Beckenham, Kent BR3 1NP, UK

e-mail: htwe@hotmail.com surae to exert over a longer rigid lever arm to promote efficient toe-off [7]. Dysfunction of the tendon results in unlocking of the talonavicular joint, allowing the triceps surae to act more proximally at the transverse tarsal joints. Abnormal loading of these joints create excessive biomechanical stresses that lead to midfoot collapse and forefoot abduction with lateral peritalar subluxation of the navicular [7]. Loss of the main invertor leaves its antagonist, the peroneus brevis, unopposed to evert the foot. The pull of the Achilles tendon falls lateral to the axis of the subtalar joint creating excessive hindfoot valgus. Progressive tightness of the triceps surae perpetuates the valgus deformity and accentuates the imbalance between the medial and lateral soft tissues. With time, the static stabilizers of the medial longitudinal arch become attenuated. The spring ligament complex is the most frequently torn static stabilizer in AAFD [15]. It supports the plantar-medial aspect of the talar head and, along with the plantar fascia and long plantar ligament, helps maintain the congruent bony arches of the foot. The superomedial fibres of the spring ligament are confluent with the anterior portion of the deltoid ligament [15]. The deltoid ligament prevents external rotation and abduction of the talus within the ankle mortise [26]. It is a static stabilizer of the tibiotalar, subtalar and talonavicular joints, as well as the spring ligament [4]. Prolonged hindfoot valgus deformity increases tension on the deltoid ligament and the medial collateral complex, causing lengthening and eventual insufficiency [4, 50]. Ultimately, these changes lead to degenerative arthrosis of the midfoot and hindfoot.

\section{Presentation}

The clinical presentation of AAFD can range from a flexible deformity with normal joint anatomy to a rigid 
deformity with advanced arthritis. The patient may develop medial pain over the posterior tibial tendon or lateral pain from subfibular impingement. Clinical signs of PTTD include hindfoot valgus, arch collapse, forefoot abduction with "too-many-toes" sign and inability to perform double and single heel-rise tests. Gastrocnemius tightness should be assessed using Silfverskiöld's test [52]. The primary clinical determinant dictating surgical management of AAFD is whether the deformity is flexible or fixed. It is then necessary to consider either a joint preservation or joint sacrificing procedure. Factors affecting this decision include patient age, function, underlying pathology, degree of deformity, flexibility, soft tissues status, body mass index and the presence of arthritis [17, 22].

\section{Classification}

The classification of PTTD has evolved over the last 20 years with further detail in categorization of the condition in order to clarify treatment recommendations. Johnson and Strom [30] described three stages of PTTD, with a fourth stage subsequently added by Myerson [40]. Stage I consists of a painful synovitis but no deformity as the tendon length and function are normal. Stage II describes progressive failure of the tendon with a flexible flatfoot deformity, which is passively correctable. In stage III, the deformity is rigid with degenerative changes in the midfoot and hindfoot. Finally, stage IV describes valgus tilting of the talus within the ankle mortise with associated deltoid insufficiency, with or without tibiotalar arthritis. In 2007, Bluman et al. [5] divided the four main stages into substages with subtle differences in clinical and radiographic findings, further demonstrating that PTTD is a wide spectrum of deformities not fully encompassed by the four-stage classification system. More recently, Parsons et al. [46] subdivided stage II disease with respect to the severity and flexibility of forefoot supination when the hindfoot is brought into neutral position (Table 1). The authors emphasized the need for early diagnosis of stage II disease in order to initiate treatment while the deformity is relatively mild and flexible.

Table 1 Truro modification of Johnson and Strom's stage II posterior tibial tendon dysfunction [46]

\begin{tabular}{lllll}
\hline Sub-stage & Deformity & Hindfoot & Forefoot supination & Correction \\
\hline IIA & Mild & Mobile & Mild $<15^{\circ}$ & Complete \\
IIB & Moderate & Mobile & Moderate $>15^{\circ}$ & Complete \\
IIC & Severe & Mobile & Severe $>15^{\circ}$ & Incomplete \\
\hline
\end{tabular}

\section{Non-operative treatment}

Advances in bracing and orthotics reveal a greater understanding of the complex deforming forces present in AAFD. Approximately $80 \%$ of early PTTD (stage I and IIA/B) respond well to non-operative management [3]. The biomechanical rationale is to alleviate forces transmitted through the posteromedial hindfoot, thereby preventing medial collapse of the foot and ankle. The patient is encouraged to lose weight, modify loading activities and wear supportive shoes. Footwear modification includes extra-depth shoes, soft leather uppers to accommodate insoles, a high toe box and a cushioned, rocker bottom to reduce ground reaction forces and assist in toe-off [45]. A custom-made, semirigid orthosis is useful in stage I or early stage II disease. The ideal design includes a deep heel cup, medial hindfoot and forefoot posting and correction of the medial longitudinal arch. A more rigid University of California Berkeley Laboratory (UCBL) orthosis is used to hold the subtalar joint in a neutral position and alleviate subfibular impingement pain [45]. In stage III disease, when custom-made or UCBL orthoses are no longer capable of stabilizing the deformity, an ankle foot orthosis (AFO) can be useful. The AFO stabilizes the medial-lateral movement of the ankle by using the leg as an anchor and limits excursion of the PTT by preventing plantarflexion and pronation of the foot [45]. Modern, low-profile designs in AFO allow control of the deformity, reduce oedema and protect the soft tissues, whilst increasing long-term compliance $[2,22,45]$. Immobilization in either a short-leg cast or a fitted removable walking boot can eliminate symptoms in stage I/II disease by reducing inflammation and oedema. It is indicated in acute tenosynovitis of the PTT, presenting with pain along the tendon sheath. The patient is advised to mobilize non-weight bearing if there is continued pain in the cast or boot. Immobilization of the foot requires a period of approximately 6-8 weeks, with concurrent rest and elevation. An adequate course of non-steroidal antiinflammatory medication is recommended. After immobilization, a custom foot orthosis, UCBL orthosis or AFO should be applied. Additionally, the patient may be sent for physical therapy for eccentric gastrocsoleus stretching exercises [1,33].

\section{Operative treatment of stage I disease}

In stage I, treatment is typically conservative but may include open or endoscopic tendon debridement [44, 54], tenosynovectomy [41, 57] and calcaneal osteotomy [62]. Funk et al. [20] reviewed nine patients undergoing tenosynovectomy with or without repair of a longitudinal tear in the posterior tibial tendon. All patients showed 
subjective and objective improvement. Pain was absent or minor in 8 of 9 patients, and these patients were capable of performing a single heel-rise postoperatively. Teasdall and Johnson [57] presented 19 patients undergoing surgical tenosynovectomy for stage I disease. Fourteen patients (74\%) reported complete pain relief, with three $(16 \%)$ experiencing minor postoperative pain. Sixteen patients (84\%) could perform a single heel-rise postoperatively. Two patients (10\%) required a subtalar arthrodesis for progressive flatfoot deformity. The benefits of endoscopic tenosynovectomy for PTTD have been reported in the short-term follow-up period. Niek van Diek et al. [44] studied six patients undergoing endoscopy for tenosynovitis and found that five were free of symptoms at an average of 1.1 year, and patients were walking comfortably in regular shoes by the 14th-postoperative day. In stage I disease, exploration and debridement, with or without FDL tendon transfer, is a viable option. In stage II disease, the PTT becomes elongated, and the medial soft tissues become attenuated. Exploration and debridement of the PTT is performed, but frequently a FDL tendon transfer or side-to-side anastomosis is required [55]. Combined procedures, including soft tissue reconstructions to restore PTT function and bony procedures to correct deformity, have become popular.

\section{Operative treatment of stage II disease}

The aim of the treatment for a flexible flatfoot deformity (stage IIA/B) is to avoid progression to a fixed deformity. Operative management options can be broadly divided into either soft tissue or bony, and the latter, into medial or lateral column procedures. There remains considerable controversy with regard to which technique or combination of techniques will give the best outcome in this subgroup. In 2003, Hiller and Pinney [25] presented the result of a survey of 104 academic foot and ankle surgeons in the United States, $98 \%$ of whom employed a soft tissue procedure as part of their surgical treatment, with $86 \%$ preferring to augment the PTT with an FDL tendon transfer. Fifty-three per cent of surgeons would formally repair the spring ligament, while $7 \%$ would repair the deltoid ligament. Of the $70 \%$ that corrected the equinus contracture, $36 \%$ performed a tendo-Achilles lengthening (TAL), $23 \%$ performed a gastrocnemius recession, and $12 \%$ performed either of these procedures depending on the clinical examination. Ninety-seven per cent of the respondents reported that they would perform some form of bony correction in their surgical protocol. Eighty-eight per cent elected to preserve the subtalar and talonavicular joints. These procedures included a medializing calcaneal osteotomy (MCO) in 73\%, lateral column lengthening (LCL) in
$41 \%$, and medial column stabilization (first TMT joint and/ or navicular-cuneiform joint arthrodesis) in $15 \%$. Of the $12 \%$ of surgeons who chose to perform arthrodesis surgery, the majority elected to fuse the subtalar joint alone. The most common combination of surgical options, reported by $38 \%$ of surgeons, was an MCO combined with some form of PTT augmentation. The second most common combination was the so-called "All-American" approach of MCO, LCL and PTT augmentation.

\section{Flexor digitorum longus transfer}

Patients undergoing an FDL transfer should have symptomatic PTTD with no or mild flatfoot deformity (stage I or early II). Proponents of the FDL tendon transfer argue that although the PTT is 3.5 times stronger, the function of the transferred FDL is to oppose the peroneus brevis, which is only 1.5 times stronger [34, 53]. Mann et al. [34-36] popularized the use of the FDL tendon transfer to augment the PTT. They concluded that although this procedure did well to relieve pain and improve inversion strength, correction of the deformity was moderate $[20,23,34,36]$. Guyton et al. [23] presented medium-term results of 26 patients treated with FDL tendon transfer with medialising calcaneal osteotomy for stage II PTTD. At a mean followup of 32 months, $91 \%$ of patients rated pain relief as good or excellent, and all but three could perform a single-leg heel rise, a manoeuvre none could perform preoperatively. Although improvement in all radiographic parameters was seen, $50 \%$ of patients felt their foot shape had not changed, and only one felt the improvement in arch height to be significant. Concerns over the lack of deformity correction with soft tissue procedures alone led many to favour additional bony realignment to protect the tendon transfer $[23,28,54]$. Myerson highlighted the importance of excising the diseased posterior tibial tendon or else pain will often remain [41, 42]. Mann [34] and others [20, 54] believed that leaving the degenerate portion of the tendon creates enough ongoing reaction that results in scarring and persistent synovitis around the tendon transfer. The degenerate PTT is released from its insertion into the navicular and cut proximally at the level of the medial malleolus, allowing it to retract proximally into the leg.

\section{Medialising calcaneal osteotomy}

The medialising calcaneal osteotomy (MCO) is useful in conjunction with an FDL tendon transfer in advanced stage I and stage II PTTD. MCO is indicated in patients in whom the valgus hindfoot deformity is passively correctable, and the pull of the Achilles tendon can be directed closer to the normal long axis of the calcaneus to the ground, in order to protect the medial tendon transfer [62]. This osteotomy is 
also effective in relieving subfibular impingement pain. In 2004, Myerson et al. [42] reported their results on 129 patients treated for PTTD with medial translational calcaneal osteotomy in conjunction with FDL tendon transfer. At a mean follow-up of 5.2 years, $91 \%$ of patients were satisfied, with statistically significant improvement in radiographic parameters measured at 6 months $(97 \%)$ and at final follow-up (87\%). One hundred and twenty-five out of 129 patients experienced pain relief, and 121 showed improvement in function. It has been hypothesized that the medialising calcaneal osteotomy corrects the flatfoot deformity by re-tensioning the plantar fascia, similar to the windlass mechanism. However, Horton et al. demonstrated in cadaveric models that the plantar fascia actually loosens after both MCO and LCL [28].

\section{Spring ligament reconstruction}

Reconstruction of the spring ligament complex corrects the flatfoot deformity in cadaveric studies but has not been proven clinically [16, 60]. Mann [34] stated that plication of the spring ligament itself does not improve the results of an FDL transfer. Sitler and Bell [54] suggested that the degeneration of ligaments during the progression of the deformity in PTTD prevents them from holding a repair, and therefore, emphasized the need for reconstruction. Choi et al. [9] used a peroneus longus tendon graft to successfully reconstruct the superomedial and inferior calcaneonavicular ligaments of the spring ligament in cadavers. The authors believed that harvesting of the peroneus longus tendon would not contribute to the flatfoot deformity, because it is left attached to the first metatarsal and tenodesed to the navicular and calcaneus, thereby avoiding dorsiflexion of the first metatarsal. However, this method has not been tested clinically.

\section{Tibialis anterior tendon procedures}

Young's tenosuspension procedure was originally described in 1939 [63]. Classically, it involved re-routing the anterior tibial tendon (ATT) into a "keyhole" in the navicular bone [29, 63]. It is performed in combination with Achilles tendon lengthening or gastrocnemius recession and is usually an adjunct to a LCL, MCO or medial column stabilization procedures. A local modification of Young's procedure involves developing a plantar grove in the navicular and securing the mobilized ATT with a bone anchor and suturing the overlying periosteum. Supination and dorsiflexion may be required for successful placement of the tendon, and care must be taken to prevent tendon rupture or navicular fracture. The effect of re-routing the ATT to the plantar aspect of the navicular results in postoperative increased forefoot supination. Jacobs [29] commented that this settles in most patients, with time and appropriate orthotic support. However, there is no clinical study to demonstrate this, and evidence remains anecdotal. El-Tayeby [18] described plantar re-routing of the tibialis anterior tendon to treat 19 paediatric severe flexible flatfeet, in conjunction with an LCL, a navicular-cuneiform medial-plantar closing wedge arthrodesis, plication of the lengthened plantar ligament and Z-plasty of the tendoAchilles. Eighty-nine per cent of patients were satisfied with the pain relief, shoe wear improvement, foot shape and general activity.

The use of the medial slip of the tibialis anterior tendon re-routed through the first cuneiform to the proximal stump of the PTT was described by Cobb and popularized by Helal [24]. Knupp and Hintermann [32] reported 22 patients with stage II PTTD and a supple supination deformity of the forefoot undergoing a Cobb procedure. Eleven patients also had a medial displacement calcaneal osteotomy, 3 had a lengthening osteotomy, 17 had repair of the deltoid, and 3 had the spring ligament repaired. At a mean follow-up of 24 months, the overall clinical results were excellent in nine patients $(41.0 \%)$, good in twelve $(54.5 \%)$, fair in one $(4.5 \%)$, and poor in none. None of the patients had decreased power of the anterior tibial tendon compared to the contralateral foot. The authors concluded that their results were comparable to those reported for medial displacement calcaneal osteotomy with flexor tendon transfer. Parsons et al. [46] prospectively assessed patient function and satisfaction after a modified Cobb procedure combined with a translational calcaneal osteotomy in 32 patients with early flexible deformity who failed conservative treatment. The modified surgery involves a bone tunnel in the navicular rather than the medial cuneiform, and re-routing the ATT behind the medial malleolus. The average follow-up was 5.1 years, and the results showed 29 out of 32 patients could perform a single heel rise at 12 months, and the mean postoperative American Orthopaedic Foot and Ankle Score (AOFAS) was 89. These observations suggest that techniques involving rerouting of the anterior tibial tendon are comparable methods of treating early stage II PTTD.

\section{Medial column bony procedures}

FDL tendon transfer combined with a medialising calcaneal osteotomy may not adequately correct the forefoot supination of the flatfoot deformity. In such cases, medial column stabilization procedures may be performed to plantarflex the medial ray [56]. The loss of the medial longitudinal arch may occur at the talonavicular, navicularcuneiform, first TMT joint or at more than one of these sites. Surgical stabilization for symptomatic medial column instability involves selective arthrodesis, including isolated 
fusion of the first TMT joint [59], navicular-cuneiform joint [64] and more extensive midfoot fusions. The opening medial cuneiform osteotomy, or Cotton osteotomy [14], has recently regained popularity in the management of residual forefoot supination following corrective hindfoot procedures for PTTD. The advantages of this osteotomy include preservation of metatarsal-cuneiform joint motion and a reliable union rate [56]. However, there are no studies to date demonstrating the long-term clinical outcome of patients undergoing this procedure, or directly comparing it with other medial column stabilization procedures. Contraindications to the Cotton osteotomy include gross metatarsal-cuneiform instability or arthritis.

Chi et al. [8] reviewed the results of medial column stabilization, lateral column lengthening and combined medial and lateral procedures in the treatment of PTTD. Four out of five feet with midfoot sag but no hindfoot valgus improved both on clinical and radiological assessment at 1-4-year follow-up after undergoing isolated medial column fusion with an FDL transfer and heel cord lengthening. Forty-eight feet with midfoot sag and associated hindfoot valgus had medial column stabilization combined with lateral column lengthening, FDL transfer and heel cord lengthening. Eighty-eight per cent of cases were pain free or had a decrease in pain at the latest followup. Toolan et al. [61] showed a similar rate of improvement in 41 feet undergoing medial column arthrodesis (first TMT and/or navicular-cuneiform joint) combined with LCL, FDL transfer and heel cord lengthening. However, they reported a high complication rate, mainly non-union at the calcaneocuboid joint and sural nerve injury during LCL. Despite this, these clinical studies demonstrate the usefulness of medial column fusions in the surgical management of stage II PTTD. It seems likely that passive correction of the hindfoot may be achieved when medial column stabilization procedures are combined with a medial soft tissue reconstruction. Additionally, Coetzee and Hansen [10] recommended medial column fusion for stage III PTTD if there is residual forefoot supination after a triple arthrodesis.

\section{Lateral column bony procedures}

The most common lateral column procedures are an Evans lateral column lengthening through the distal calcaneus [19, 39, 51] or a calcaneocuboid distraction arthrodesis (CCDA) [39, 58]. LCL is used in conjunction with medial soft tissue balancing and, if appropriate, gastrocnemius recession [11, 27, 59]. The aim of the LCL is to adduct the forefoot at the talonavicular joint and correct the peritalar subluxation. The Evans technique involves placement of a tri-cortical iliac crest or allograft into the distal calcaneal osteotomy made parallel and $10-15 \mathrm{~mm}$ proximal to the calcaneocuboid joint (CCJ). The graft is held in place by a screw or a low-profile plate [21, 51]. Lateral column lengthening adequately corrects forefoot abduction, but it does not reliably correct the medial deformity in the sagittal plane. A trapezoid-shaped graft in the osteotomy site creates forefoot adduction as well as plantarflexion, preventing excessive forefoot supination and symptomatic lateral column overload [43]. Evans [19] proposed that the windlass mechanism was responsible for the restoration of the medial longitudinal arch during LCL. This idea of a bowstringing effect within the plantar fascia, long plantar ligament and peroneus longus tendon is supported by other authors [38, 48]. However, Horton et al. [28] demonstrated the opposite effect with loss of tension in the plantar fascia after performing LCL. Morphological assessment of the talar head shows that its height is less than its width. As the lateral column is lengthened, the navicular is translated medially on the talar head. There is also plantar translation on the talar head, as this is the smaller radius of curvature and therefore the shortest distance [21]. This results in plantarflexion of the forefoot at the talonavicular joint and elevation of the medial longitudinal arch.

An anatomical study by Raines and Brage [49] confirmed the ideal site for an Evans osteotomy to be $10 \mathrm{~mm}$ proximal to the CCJ, between the anterior and middle facets of the subtalar joint. A more proximal osteotomy risks injury to the medial-plantar nerve, long flexor tendons and tibialis posterior tendon. The more distal osteotomies endangered the sural nerve and peroneal tendons, as well as risk breakthrough of pins into either the osteotomy site or CCJ [49, 51]. Most authors advocate the site of the Evans osteotomy to be 10-15 mm proximal to the joint $[19,48,51]$, while the length of distraction ranges from 8 to $14 \mathrm{~mm}[21,27,31,39$, 48]. The ideal size of distraction, and hence bone graft, depends on intra-operative clinical and fluoroscopic assessment of adequate talar head coverage and return of the medial longitudinal arch. A randomized study by Dolan and colleagues investigated the type of graft used to lengthen the lateral column. There was no difference found between allograft and autograft in the rate of non-union or graft collapse in 33 cases. The rate of non-union with lateral column lengthening through CCDA is higher than an Evans calcaneal lengthening [12, 39, 61]. Non-union rate is quoted as $1-5 \%$ with increased risk in smokers [6, 39, 43]. A criticism of LCL is that it does not correct the deforming force of the Achilles tendon resulting in continued hindfoot valgus. An additional medialising calcaneal osteotomy can correct hindfoot valgus and alter the direction of pull from the Achilles tendon from eversion to a neutral axis.

The development of calcaneocuboid arthritis has been an area of concern following lateral column lengthening with the theoretical risk of increasing joint pressures. However, the evidence for this is conflicting. Cooper et al. 
[13] stated that CCJ pressures increased eightfold after $10 \mathrm{~mm}$ lengthening of the lateral column, while Momberger et al. [37] showed no difference in pressures in cadaveric planovalgus models. The actual clinical risk of CCJ arthritis after LCL is also debated. Phillips [47] found 15 out of 23 feet had radiographic evidence of CCJ arthrosis at 13 years after an Evans procedure. Hintermann et al. [27] found only 2 out of 19 patients at an average follow-up of 23 months developed degenerative changes in the CCJ, with only one patient requiring arthrodesis.

In 2007, Bolt et al. [6] compared 25 LCL with 17 MCO. The initial correction of the tarsometatarsal angle was three times greater in the LCL group. Realignment of the medial column and medial longitudinal arch were also superior with LCL. The authors also demonstrated a greater subsequent loss of correction with MCO compared to LCL at final follow-up. Thomas et al. [58] compared an Evans LCL in 10 feet with CCDA in 17 feet. Both groups underwent PTT debridement, FDL tendon transfer and use of iliac crest graft. Both procedures produced significant improvement in radiological parameters and AOFAS scores, but no significant difference was seen between the two groups. However, the authors noted a high complication rate with both techniques, and the rate of non-union and delayed union with calcaneocuboid distraction arthrodesis remained a major problem.

In conclusion, LCL is probably best performed through the distal calcaneus rather than CCJ distraction arthrodesis. A concomitant medialising calcaneal osteotomy and/or medial soft tissue rebalancing is recommended. A trapezoid-shaped bone graft can be used in order to avoid excessive forefoot supination. The correction of forefoot abduction is greater with LCL than medial column procedures, but the rates of complications are higher. Correction of any gastrocnemius tightness is paramount.

\section{Conclusion}

Posterior tibial tendon dysfunction is the most common cause of adult acquired flatfoot deformity. The main deforming forces, tendo-Achilles and peroneus brevis, act to promote hindfoot eversion and attenuate structures that stabilize the medial column. More detailed classification systems have been developed in recent years to help clarify treatment recommendations. The commonest surgical management of stage II PTTD is medialising calcaneal osteotomy with FDL tendon transfer. Techniques to restore medial column stability in adult acquired flatfoot have evolved in the last two decades. In addition to arthrodesis procedures of the first ray, an opening wedge (Cotton) osteotomy of the medial cuneiform is now a popular alternative and preserves joint motion. The opening wedge osteotomy forces the medial column into slight plantarflexion, tightening the plantar fascia and adding stability to the first tarsometatarsal and navicular-cuneiform joints. Arthrodesis of these joints is warranted only when arthritis or instability is present.

Correcting forefoot abduction deformity has been pivotal to the development of medial and lateral column procedures. When the forefoot is abducted, there is increased uncovering of the talonavicular joint, and the lateral column becomes relatively short compared to the medial column. Lengthening of the calcaneocuboid joint with arthrodesis has a higher rate of complication, but lengthening of the anterior calcaneus with an osteotomy proximal to the joint avoids the risk of non-union inherent in a calcaneocuboid distraction arthrodesis. Furthermore, combination procedures are now more prevalent such as lengthening of the lateral column with simultaneous plantarflexion procedures of the medial column and/or medial soft tissue augmentation. The medial column procedures off-load the lateral column lengthening and prevent symptomatic overload of the lateral column. These combination procedures emphasize the need for careful rebalancing of medial and lateral deforming forces in the surgical correction of a flexible adult acquired flatfoot deformity.

\section{References}

1. Alvarez RG, Marini A, Schmitt C, Saltzman CL (2006) Stage I and II posterior tibial tendon dysfunction treated by a structured nonoperative management protocol: an orthosis and exercise program. Foot Ankle Int 27:2-8

2. Augustin JF, Lin SS, Berberian WS, Johnson JE (2003) Nonoperative treatment of adult acquired flat foot with the Arizona brace. Foot Ankle Clin 8:491-502

3. Barrie J (2009) Symposium on the adult flat foot: orthotic management. British orthopaedic foot \& ankle society annual scientific meeting, Old Windsor, 4-6th November 2009

4. Bluman EM, Myerson MS (2007) Stage IV posterior tibial tendon rupture. Foot Ankle Clin 12:341-362

5. Bluman EM, Title CI, Myerson MS (2007) Posterior tibial tendon rupture: a refined classification system. Foot Ankle Clin 12:233249

6. Bolt P, Coy S, Toolan B (2007) A comparison of lateral column lengthening and medial translational osteotomy of the calcaneus for the reconstruction of adult acquired flat foot. Foot Ankle Int 28:1115-1123

7. Brodsky JW (2004) Preliminary gait analysis results after posterior tibial tendon reconstruction: a prospective study. Foot Ankle Int 25:96-100

8. Chi T, Toolan B, Sangeorzan B, Hansen ST Jr (1999) The lateral column lengthening and medial stabilisation procedures. Clin Orthop Relat Res 365:81-90

9. Choi K, Lee S, Otis JC et al (2003) Anatomical reconstruction of the spring ligament using peroneus longus tendon graft. Foot Ankle Int 24:430-436

10. Coetzee JC, Hansen ST Jr (2001) Surgical management of severe deformity resulting from posterior tibial tendon dysfunction. Foot Ankle Int 22:944-949 
11. Coetzee JC, Castro MD (2003) The indications and biomechanical rationale for various hindfoot procedures in the treatment of posterior tibialis tendon dysfunction. Foot Ankle Clin 8:453-459

12. Conti SF, Wong YS (2002) Osteolysis of structural autograft after calcaneocuboid distraction arthrodesis for stage II posterior tibial tendon dysfunction. Foot Ankle Int 23:521-529

13. Cooper P, Nowak M, Shaer J (1997) Calcaneocuboid joint pressures with lateral column lengthening (Evans) procedure. Foot Ankle Int 18:199-205

14. Cotton FJ (1936) Foot statistics and surgery. N Engl J Med 214:353-362

15. Deland JT (2001) The adult acquired flatfoot and spring ligament complex. Pathology and implications for treatment. Foot Ankle Clin 6:129-135

16. Deland JT, Arnoczky SP, Thompson FM (1992) Adult acquired flat foot deformity at the talonavicular joint: reconstruction of the spring ligament in an in vitro model. Foot Ankle 13:327-332

17. Elftman NW (2003) Nonsurgical treatment of adult acquired flat foot deformity. Foot Ankle Clin 8:473-489

18. El-Tayeby HM (1999) The severe flexible flatfoot: a combined reconstructive procedure with rerouting of the tibialis anterior tendon. J Foot Ankle Surg 38:41-49

19. Evans D (1975) Calcaneovalgus deformity. J Bone Joint Surg Br 57:270-278

20. Funk DA, Cass JR, Johnson KA (1986) Acquired adult flatfoot secondary to posterior tibial tendon pathology. J Bone Joint Surg Am 68:95-102

21. Gallina J, Sands AK (2003) Lateral-sided bony procedures. Foot Ankle Clin 8:563-567

22. Giza E, Cush G, Schon LC (2007) The flexible flatfoot in the adult. Foot Ankle Clinics 12:251-271

23. Guyton GF, Jeng C, Krieger LE, Mann RA (2001) Flexor digitorum longus transfer and medial displacement calcaneal osteotomy for posterior tibial tendon dysfunction: a middle-term clinical follow-up. Foot Ankle Int 22:627-632

24. Helal B (1990) Cobb repair for tibialis posterior tendon rupture. J Foot Surg 29:349-352

25. Hiller L, Pinney SJ (2003) Surgical treatment of acquired flatfoot deformity: what is the state of practice among academic foot and ankle surgeons in 2002? Foot Ankle Int 24:701-705

26. Hintermann B (1999) Biomechanics of the unstable ankle joint and clinical implications. Med Sci Sports Exerc 31:459-469

27. Hintermann B, Valderrabano V, Kundert HP (1999) Lengthening of the lateral column and reconstruction of the medial soft tissue for the treatment of acquired flat foot deformity associated with insufficiency of the posterior tibial tendon. Foot Ankle Int 20:622-629

28. Horton GA, Myerson MS, Parks BG, Park YW (1998) Effect of calcaneal osteotomy and lateral column lengthening on the plantar fascia: a biomechanical investigation. Foot Ankle Int 19:370-373

29. Jacobs AM (2007) Soft tissue procedures for the stabilization of medial arch pathology in the management of flexible flatfoot deformity. Clin Podiatr Med Surg 24:657-665

30. Johnson KA, Strom DE (1989) Tibialis posterior tendon dysfunction. Clin Orthop Relat Res 239:196-206

31. Kelly IP, Easley ME (2001) Treatment of stage 3 adult acquired flatfoot. Foot Ankle Clin 6:153-166

32. Knupp M, Hintermann B (2007) The Cobb procedure for treatment of acquired flatfoot deformity associated with stage II insufficiency of the posterior tibial tendon. Foot Ankle Int 28:416-421

33. Kulig K, Lederhaus ES, Reischl S, Arya S, Bashford G (2009) Effect of eccentric exercise program for early tibialis posterior tendinopathy. Foot Ankle Int 30:877-885
34. Mann RA (2001) Posterior tibial tendon dysfunction: treatment by flexor digitorum longus transfer. Foot Ankle Clin 6:77-87

35. Mann RA (1983) Acquired flat foot in adults. Clin Orthop Relat Res 181:46-51

36. Mann RA, Thompson FM (1985) Rupture of the posterior tibial tendon causing flat foot. J Bone Joint Surg Am 67:556-561

37. Momberger N, Morgan J, Bachus K, West J (2000) Calcaneocuboid pressure after lateral column lengthening in a cadaveric planovalgus deformity model. Foot Ankle Int 21:730-735

38. Mosca VH (1995) Calcaneal lengthening for valgus deformity of the hindfoot. Results in children who had severe, symptomatic flatfoot and skewfoot. J Bone Joint Surg Am 77:500-552

39. Mosier-LaClair S, Pomeroy G, Manoli A (2001) Operative treatment of the difficult stage 2 adult acquired flat foot deformity. Foot Ankle Clin 6:95-119

40. Myerson MS (1997) Adult acquired flatfoot deformity: treatment of dysfunction of the posterior tibial tendon. Instr Course Lect 46:393-405

41. Myerson M, Solomon G, Shereff M (1989) Posterior tibial tendon dysfunction: Its association with seronegative inflammatory disease. Foot Ankle Int 9:219-225

42. Myerson MS, Badekas A, Schon LC (2004) Treatment of stage II posterior tibial tendon deficiency with flexor digitorum longus tendon transfer and calcaneal osteotomy. Foot Ankle Int 25:445450

43. Neufield SK, Myerson M (2001) Complications of surgical treatments for adult flat foot deformities. Foot Ankle Clin 6:179 192

44. Niek van Diek C, Kort N, Scholten PE (1997) Tendonoscopy of the posterior tibial tendon. Arthroscopy 13:692-698

45. Noll KH (2001) The use of orthotic devices in adult acquired flatfoot deformity. Foot Ankle Clin 6:25-36

46. Parsons S, Naim S, Richards PJ, McBride D (2009) Correction and prevention of deformity in type II tibialis posterior dysfunction. Clin Orthop Relat Res Oct 22 [Epub ahead of print]

47. Phillips GE (1983) A review of elongation of os calcis for flat feet. J Bone Joint Surg Br 65:15-18

48. Pomeroy G, Manoli A (1997) A new operative approach for flat foot secondary to posterior tibial tendon insufficiency: a preliminary report. Foot Ankle Int 18:206-212

49. Raines R, Brage M (1998) Evans osteotomy in the adult foot: an anatomical study of structures at risk. Foot Ankle Int 19:743-747

50. Resnick RB, Jahss MH, Choueka J, Kummer F, Hersch JC et al (1995) Deltoid ligament forces after tibialis posterior tendon rupture: effects of triple arthrodesis and calcaneal displacement osteotomies. Foot Ankle Int 16:14-20

51. Sands AK, Tansey JP (2007) Lateral column lengthening. Foot Ankle Clin 12:301-308

52. Silfverskiöld N (1924) Reduction of the uncrossed two-joints muscles of the leg to one-joint muscles in spastic conditions. Acta Chir Scand 56:315-328

53. Silver RL, DeLaGarza J, Rang N (1985) The myth of muscle balance: a study of relative strengths and excursions of normal muscles about the foot and ankle. J Bone Joint Surg Br 67:432-437

54. Sitler DF, Bell SJ (2003) Soft tissue procedures. Foot Ankle Clin $8: 503-520$

55. Sizensky JA, Marks RM (2003) Medial-sided bony procedures: why, what, and how? Foot Ankle Clin 8:539-562

56. Tankson CJ (2007) The cotton osteotomy: indications and techniques. Foot Ankle Clin 12:309-315

57. Teasdall RD, Johnson KA (1994) Surgical treatment of stage I posterior tibial tendon dysfunction. Foot Ankle 15:646-648

58. Thomas RT, Wells BC, Garrison RL, Prada SA (2001) Preliminary results comparing two methods of lateral column lengthening. Foot Ankle Int 22:107-119 
59. Thompson IM, Bohay DR, Anderson JG (2005) Fusion rate of first tarsometatarsal arthrodesis in the modified Lapidus procedure and flatfoot reconstruction. Foot Ankle Int 26:698-703

60. Thordarson DB, Schmotzer H, Chon J (1995) Reconstruction with tenodesis in an adult flat foot model. J Bone Joint Surg Am 77:1557-1564

61. Toolan BC, Sangeorzan BJ, Hansen ST Jr (1999) Complex reconstruction for the treatment of dorsolateral peritalar subluxation of the foot. J Bone Joint Surg Am 81:1545-1560
62. Weinfeld SB (2001) Medial slide calcaneal osteotomy: technique, patient selection, and results. Foot Ankle Clin 6:89-94

63. Young CS (1939) Operative treatment of pes planus. Surg Gynecol Obstet 99:1099-1101

64. Younger A, Wing K (2003) Medial column fusion for type 2 posterior tibial tendon deficiency: the navicular cuneiform fusion to correct forefoot varus. J Foot Ankle Surg 2:74-83 